\title{
Philosophiques
}

\section{Un retournement dans la philosophie de la biologie de K.R. Popper}

\section{François Tournier}

Volume 18, numéro 1, printemps 1991

URI : https://id.erudit.org/iderudit/027141ar

DOI : https://doi.org/10.7202/027141ar

Aller au sommaire du numéro

Éditeur(s)

Société de philosophie du Québec

ISSN

0316-2923 (imprimé)

1492-1391 (numérique)

Découvrir la revue

Citer cet article

Tournier, F. (1991). Un retournement dans la philosophie de la biologie de K.R. Popper. Philosophiques, 18(1), 61-94. https://doi.org/10.7202/027141ar
Résumé de l'article

La littérature épistémologique actuelle véhicule une image caricaturale de la philosophie popperienne de la biologie. En effet, on suppose sa position suffisamment claire et univoque pour pouvoir se résumer succinctement en quelques lignes. De plus, on la suppose toujours la même tout au long de l'évolution intellectuelle de son auteur. Dans le présent article, nous voudrions contester ces deux suppositions car sa pensée est non seulement vague et ambiguë mais encore elle est loin d'être constante et homogène. De ce fait, nous entendons corriger une conception eronnée et généralement répandue de sa philosophie de la biologie. 
PHILOSOPHIQUES, Vol. XVIII, Numéro 1, Printemps 1991

\title{
UN RETOURNEMENT DANS LA PHILOSOPHIE DE LA BIOLOGIE DE K.R. POPPER**
}

\author{
par François Tournier
}

RÉsUMÉ. La littérature épistémologique actuelle véhicule une image caricaturale de la philosophie popperienne de la biologie. En effet, on suppose sa position suffisamment claire et univoque pour pouvoir se résumer succinctement en quelques lignes. De plus, on la suppose toujours la même tout au long de l'évolution intellectuelle de son auteur. Dans le présent article, nous voudrions contester ces deux suppositions car sa pensée est non seulement vague et ambiguë mais encore elle est loin d'être constante et homogène. De ce fait, nous entendons corriger une conception eronnée et généralement répandue de sa philosophie de la biologie.

ARSTRACT. Popper's philosophy of biology is generally misconceived. It is supposed to be homogeneous and clearly statable in a few lines. Worse, Popper's changes of mind on these questions are not even taken into account. In this article, we will try to show why these suppositions are erroneous and how they hinder us from forming a more adequate idea of his philosophy of biology.

** Je voudrais remercier M. Paul Fournier, étudiant au doctorat en biologie à l'Université Laval, le prof. Michel Delsol (Faculté Catholique des Sciences de l'Universite de Lyon), le prof. Robert Nadeau (Université du Québec à Montrél), le prof. Thomas De Koninck (Université Laval) et le prof. J.N. Kaufmann (Université du Québec à Trois-Rivières) et les Evaluateurs de la revue Pbilosophiques pour leurs arguments et leurs précieuses suggestions de corrections qui m'ont permis d'améliorer grandement des versions antérieures de ce texte. On ne saurait cependant leur imputer les idées qui sont exprimées ici, ni la responsabilité des carences que pourrait contenir le présent texte. Je voudrais également remercier le Conseil de Recherche en Sciences Humaines du Canada dont la subvention a permis la présente recherche. 


\section{Introduction}

Le but du présent exposé est double: montrer, d'une part, qu'il y a eu un revirement dans la philosophie popperienne de la biologie et, d'autre part, que son incursion dans ce champ de recherche n'est pas sans valeur malgré ses lacunes manifestes. La patiente et minutieuse analyse nécessaire pour mettre en évidence le retournement popperien est certes intéressante en soi mais elle acquiert une importance supplémentaire du fait que la plupart des commentateurs de Popper comme R. Nadeau (1983), R. Bouveresse (1978), M. Ruse (1977), D.B. Kitts (1977) ou K.K. Lee (1969) n'en tiennent pas compte dans leurs écrits. Il importe donc de remplir ce "blanc» dans la littérature actuelle afin de se former une idée plus juste des conceptions de Popper sur la biologie évolutionniste.

La mise en évidence de la pertinence de son intervention en biologie soulève cependant une difficulté particulière. Il est nécessaire, pour pouvoir l'accepter, d'aller à l'encontre d'un idéal de la recherche généralement admis, à savoir, que pour contribuer à l'avancement d'un débat quelconque, il faut au minimum avoir une très bonne connaissance du domaine concerné. Or, de son propre aveu, Popper intervient *en amateur" dans le champ de la biologie évolutionniste. On ne trouve pas dans ses écrits des discussions sur la théorie génétique de Mendel à Crick et Watson, sur la génétique des populations de Hardy Weinberg à Fisher, Haldane et Wright, sur la théorie de l'épigénèse de Waddington ou sur la théorie de la spéciation de Mayr à Eldridge et Gould. On ne trouve pas non plus de référence aux travaux philosophiques importants et récents sur ces questions comme ceux de M. Ruse (1973), de Mary Williams (1973)(1984) ou de E. Sober (1984a) et (1984b). Les discussions de Popper traitent des problèmes «en principe" (seulement) et les exemples qu'il apporte sont le plus souvent des cas imaginaires ou des hypothèses fictives (comme la possibilité de l'apparition d'une grande variété de formes de vie sur la planète Mars). Popper (1978 : 344) avoue même qu'il a été *influencé par ce qu'ont dit les autorités » comme C.H. Waddington, Ronald Fisher, J.B.S. Haldane et G.G. Simpson sur la théorie de la sélection naturelle pour la considérer pendant longtemps comme une "quasi-tautologie». Il n'en faudra pas plus pour *décourager» les spécialistes de la biologie évolutionniste de trouver une quelconque contribution pertinente aux débats actuels chez Popper. En fait, une lecture un peu rapide et peu 
attentive de ses écrits leur laisse le plus souvent une impression défavorable assez similaire à celle qu'ils éprouvent en écoutant les propos critiques de certains étudiants impétueux qui devraient commencer par apprendre d'abord ce dont ils parlent.

Par exemple, lorsque Popper nie la possibilité de formuler des lois de l'évolution, il se trouvera plus d'un commentateur pour dénoncer sa confusion entre l'histoire de la vie sur Terre et une théorie scientifique visant à expliquer l'évolution des populations ou des espèces par subsomption sous des « lois *. De même, lorsque Popper considère comme "circulaire" une explication de l'aptitude à s'adapter en termes de survie, plusieurs dénonceront sa confusion entre l'aptitude à s'adapter et la survie qui peuvent toutes deux être définies indépendamment l'une de l'autre. Autrement dit, Popper semble commettre des confusions élémentaires qu'il aurait pu facilement éviter en étant plus au fait de la littérature actuelle sur ces questions. Mais, à trop vouloir insister sur ces distinctions, on risque fort de passer complètement à côté de ce qui constitue, comme nous tenterons de le montrer, des contributions durables de Popper aux débats épistémologiques entourant la théorie de l'évolution.

Dans les pages qui suivent, on ne trouvera pas de réponse aux diverses critiques de sa philosophie de la biologie et, notamment, à celles de M. Ruse (1977) dont l'érudition en biologie contraste étrangement avec celle de Popper. Qu'il se soit trompé sur certains points, comme il l'avoue lui-même par ailleurs, ne prouve en rien qu'il ne pourrait pas avoir raison sur d'autres points. Nous ne tenterons donc pas de contrer ces critiques maintenant courantes mais seulement de remettre en question leur supposition fondamentale, à savoir, que la position de Popper en biologie est limpide, univoque et se laisse facilement cerner - du moins assez pour pouvoir faire l'objet d'une critique décisive.

En effet, la philosophie de la biologie de K.R. Popper est dans un état si fragmentaire, éparse et obscure qu'elle prête facilement à confusion. Il est même très douteux qu'on puisse définir sa position en biologie évolutionniste d'une façon claire et univoque comme il est possible de le faire pour sa philosophie de la physique. Popper n'a pas consacré autant de temps et d'efforts à la résolution des problèmes épistémologiques soulevés par la théorie de l'évolution qu'à ceux soulevés par la physique. Il y a, pourrait-on dire, des «trous noirs» dans l'ceuvre de Popper, notamment en ce qui 
concerne sa philosophie de l'économie et sa philosophie de la biologie. Si les imprécisions et les ambiguités de sa philosophie des sciences sociales ont été largement décelées et font partie intégrante des débats actuels, il n'en va pas de même pour celles concerant sa philosophie de la biologie qui demeurent à ce jour généralement méconnues. On croit habituellement que la position de Popper est assez claire et précise pour se résumer brièvement. Pour reprendre la formulation qu'en donne R. Nadeau (1983: 5), "le darwinisme est une théorie non testable, non prédictive, ad boc, non nomologique /.../ [que] l'on serait malvenu de /.../ déclarer scientifique». Une telle interprétation suppose que les passages fragmentaires, dispersés ici et là à travers son œuvre et formulés à différentes périodes de son évolution intellectuelle, constituent une unité transparente. Mais, la lecture limpide des textes de Popper apparaît rapidement illusoire dès qu'on prend conscience d'une série de difficultés rendant la reconstruction de sa pensée des plus ardues.

Une première difficulté d'importance est l'usage terminologique inconséquent de Popper. Comme nous le verrons, il utilise la même expression "théorie de l'évolution * pour se référer à l'hypothèse évolutionniste, à l'hypothèse de la sélection naturelle, au système théorique néo-darwinien au complet ou même à la théorie originale de Darwin. Seule une attention minutieuse au contexte discursif peut nous permettre de déterminer le sens qu'il confère à cette expression dans tel ou tel passage particulier. Il n'est pas évident non plus que le terme *darwinisme * conserve toujours la même signification à travers son œuvre. Nous supposerons ici l'identité de signification car, bien que son nom varie ("théorie darwinienne", "théorie néo-darwinienne", "théorie orthodoxe", "théorie de l'évolution", "théorie synthétique", "hypothèse darwinienne*, "hypothèse évolutionniste"), il est clair que, pour Popper, le darwinisme a toujours été conçu comme un système théorique constitué de plusieurs hypothèses même si, le plus souvent, il les examine une à la fois. Il va de soi qu'une interprétation adéquate de la position de Popper en biologie ne doit pas répéter les mêmes inconséquences terminologiques et bien identifier dans chaque cas, à laquelle de ces hypothèses, prises isolément ou en groupe, le passage fait référence. Une telle exigence exclut d'emblée bien sûr les lectures hâtives et négligeantes des textes de Popper.

Une autre difficulté de taille consiste à bien distinguer les différents niveaux des interventions de Popper en biolo- 
gie évolutionniste. Ainsi, insatisfait de la façon dont la théorie néo-darwinienne explique l'apparition d'un organe aussi complexe que l'œil, c'est-à-dire par une série de petites mutations indépendantes et accidentelles, Popper (1972: 270-84 et 1974: 133-43) propose de modifier la théorie orthodoxe de façon à y intégrer une distinction entre la fonction (physiologique) d'un organe complexe et la capacité de l'organisme à s'en servir habilement pour sa survie. Selon la conjecture popperienne, l'acquisition de cette capacité est une condition préalable à l'apparition de l'organe complexe. Après tout, les manipulations appropriées d'instruments de précision très perfectionnés exigent une certaine compétence. En conséquence, l'acquisition de cette habileté préalable vient guider, bien que d'une façon très diffuse, la direction de l'évolution qui n'est pas aussi hasardeuse que ne le prétend la théorie orthodoxe. Plusieurs biologistes y verront bien sûr un retour à des hypothèses biologiques démodées mais ce qui importe pour notre propos est que cette in tervention de Popper se situe sur le plan scientifique et non sur le plan méthodologique. De même, lorsque, dans la lignée de l'empiriocriticisme de Mach et d'Avenarius (qui, comme on le sait, ont cherché à asseoir leur théorie de la connaissance sur la biologie de leur époque et plus spécifiquement sur la théorie darwinienne de l'évolution), Popper (1972) entend également fonder sa théorie de la connaissance (une généralisation de sa méthodologie scientifique) sur la théorie de l'évolution en la rendant conforme à une conception de l'être humain qui en fait un produit de l'évolution de la vie sur Terre, celui-ci intervient sur un plan métaphysique et non au niveau méthodologique. La connaissance humaine apparaît ainsi comme un moyen ou une capacité développée par l'espèce Homo Sapiens pour mieux s'adapter à son environnement. Dans cette perspective, la méthodologie falsificationniste elle-même est présentée comme le raffinement logique et conscient d'un processus déjà à l'œuvre dans la nature, à savoir, la sélection naturelle (la méthode de l'essai et de l'erreur). Une interprétation adéquate de la position de Popper doit donc bien distinguer ces niveaux d'intervention scientifique et métaphysique du niveau méthodologique dont nous ferons exclusivement notre objet dans la présente recherche.

Mais, la difficulté la plus importante à laquelle se heurte une reconstruction de la pensée de Popper sur la biologie évolutionniste est certainement le fait qu'il a changé d'idée sur certaines questions. Comme le note Eliot Sober (1984a : 61), 
Popper s'est rétracté par rapport à sa caractérisation de l'hypothèse de la survie du plus apte qu'il considérait auparavant comme une pétition de principe ou un énoncé tautologique. Popper change également d'idée, semble-t-il, par rapport à au moins deux autres hypothèses du système théorique néo-darwinien. Une interprétation adéquate de sa position doit évidemment tenir compte de ces revirements popperiens et de leurs implications. En ce sens, à l'encontre d'une supposition à la base de beaucoup de critiques de la position de Popper, un des buts du présent exposé est de montrer comment ces difficultés rendent très incertaine l'interprétation à donner de sa position. Autrement dit, les affirmations trop catégoriques et les résumés simplistes sont à éviter.

L'exemple du débat entre Halstead et Popper est particulièrement éloquent en ce qui concerne une lecture des textes popperiens qui ne tient pas compte des difficultés mentionnées plus haut. L'exemple est d'autant plus significatif que Halstead reprend pour une large part l'interprétation courante de la philosophie popperienne de la biologie - celle à la base de la majorité des critiques qui lui sont adressées. Dans un article intitulé *Popper: good pbilosophy, bad science?», Beverley Halstead (1980), un géologue et zoologiste de Reading University, entend s'opposer à l'idée de plus en plus à la mode (même parmi les biologistes!) selon laquelle l'hypothèse évolutionniste ou les hypothèses paléontologiques ne seraient pas testables et, en conséquence, seraient aussi métaphysiques et spéculatives que l'ancienne hypothèse créationniste dont il constate avec effroi le renouveau de popularité. Parmi les principaux promoteurs de cette idée de plus en plus à la mode, le Dr Halstead place au premier chef K.R. Popper dont il condamne par ailleurs la méthodologie scientifique pour avoir servi à sanctionner et à justifier une idée aussi réactionnaire. Comme nous le verrons, la théorie de l'évolution représente sans l'ombre d'un doute un «cas problème* pour sa méthodologie falsificationniste. Donc, si on interprète ces difficultés méthodologiques comme des «condamnations \# de cette théorie, les réticences du naturaliste anglais sont tout à fait compréhensibles et naturelles. Après tout, Popper intervient "en amateur" dans un secteur de la connaissance scientifique où il n'est pas particulièrement à l'aise et, prises au pied de la lettre, certaines de ses affirmations paraissent très critiquables. Cependant, et le Dr Halstead ne l'affirme pas non plus, on ne saurait l'accu- 
ser de prôner un retour à l'hypothèse périmée du Créateur universel.

Évitant le plus possible toute inférence et toute interprétation, le Dr Halstead se contente de citer textuellement des passages disséminés à travers certains de ses écrits où Popper affirme clairement que le darwinisme n'est pas une hypothèse scientifique testable, qu'elle ressemble à un programme métaphysique de recherche et que son statut épistémologique est à peu près équivalent à celui d'un récit historique - le moins scientifique des discours scientifiques. Halstead (tout comme Ruse (1977) d'ailleurs) se réfêre surtout à The Poverty of Historicism (1956), à The Logic of Scientific Discovery (1959) et à Unended Quest (1976) comme si ces écrits formaient un tout homogène. Si on considère que Logik der Forschung (l'édition allemande originale de Popper (1959)) est paru en 1934 et que Unended Quest est une adaptation sous forme de livre de son "Autobiography" paru originalement dans The Pbilosophy of K.R. Popper (1974) dont Schilpp est l'éditeur, la revue de littérature du Dr Halstead couvre une période d'environ $\mathbf{4 0}$ ans, soit de 1934 à 1974 .

Ces références à l'appui, la «surprise» du Dr Halstead a certainement dû être des plus grandes (comme devrait l'être d'ailleurs celle des partisans de l'interprétation courante de la position de Popper) de se voir formellement démenti par Popper lui-même (1980: 611) dans le numéro suivant de la revue :

/.../ certaines personnes pensent que j'ai contesté le caractère scientifique des sciences historiques comme la paléontologie ou de l'histoire de l'évolution de la vie sur Terre /.../

C'est une erreur et je voudrais affirmer ici que celles-ci, tout comme d'autres sciences historiques, ont, à mon avis, un caractère scientifique: dans bien des cas, leurs hypothèses peuvent être testées.

En fait, la critique de Halstead suppose que toutes ces références s'inscrivent dans la même perspective et dans la même ligne d'idée, et ce qu'il faut savoir c'est qu'en 1977 , lors d'une conférence au Darwin College (Cambridge) - le texte paraîtra l'année suivante dans la revue Dialectica -, Popper avoue avoir " changé d'idée à propos de la testabilité et du statut logique de la théorie de la sélection naturelle * (1978: 345). Il semble également se rétracter par rapport à l'hypothèse de l'ascendance commune puisqu'auparavant il la considérait comme un point de vue historique non testable et que maintenant il écrit (1978: 344) : 
Les fondations mendeliennes du darwinisme moderne ont été bien testées, de même que la théorie de l'évolution qui affirme que toute vie terrestre a évolué à partir de quelques organismes primitifs unicellulaires, possiblement même d'un seul organisme.

Si on ajoute à ces revirements apparents la rétractation dont parlait Sober (1984a : 61) plus haut, cela fait au moins trois hypothèses du système théorique néo-darwinien par rapport auxquelles Popper semble avoir changé d'idée : l'hypothèse de la survie des plus aptes, l'hypothèse de la sélection naturelle et l'hypothèse évolutionniste.

Afin d'éviter les confusions que nous avons mentionnées plus haut, commençons par spécifier l'objet de la présente enquête en dégageant des textes de Popper sa conception du "darwinisme".

En parlant de darwinisme ici, écrit Popper (1978: 343-4), j'entendrai toujours la théorie actuelle, c'est-à-dire la théorie originale de Darwin sur la sélection naturelle fondée sur la théorie mendelienne de l'hérédité, sur la théorie des mutations et des combinaisons génétiques et sur le décodage du code génétique.

Ensemble ces diverses hypothèses constituent « une explication d'une multitude d'observations biologiques et paléontologiques » (1956: 108). Mais c'est véritablement dans son «Autobiograpby" (Schilpp (ed.) 1974: 136-7) que Popper spécifie le plus clairement ce qu'il entend par cette expression. Son analyse logique révèle que le système théorique néo-darwinien est composé d'au moins cing hypothèses fondamentales qu'il subdivise en deux sous-groupes (S1) et (S2), selon leur fonction dans l'explication des phénomènes évolutifs : (h1) l'hypothèse évolutionniste selon laquelle la grande variété des formes de vie sur Terre a pour origine un petit nombre de telles formes, peut-être même une seule; (h2) l'hypothèse de l'hérédité avec ses fondements génétiques selon laquelle les descendants reproduisent assez fidèlement les organismes de leurs parents; (h3) l'hypothèse de la variation (ou de mutations au hasard) selon laquelle il se produit de petites variations héréditaires indépendantes et accidentelles; (h4) l'hypothèse de la sélection naturelle selon laquelle les petites variations accidentelles sont soumises à des mécanismes d'élimination ; et (h5) l'hypothèse de la survie des plus aptes selon laquelle plusieurs formes de vie contrôlées par la sélection naturelle sont en présence et luttent pour leur survie. 
Pour bien comprendre la division popperienne de ces hypothèses en deux sous-groupes (S1) et (S2), il importe de rappeler ici que Darwin et les néo-darwiniens sont à la fois des historiens et des théoriciens (pour reprendre la dichotomie introduite par Popper dans Misère de l'historicisme (1956)). Du fait que Popper distingue catégoriquement les sciences historiques des sciences théoriques en leur assignant chacune des caractéristiques épistémologiques distinctes, ce double intérêt de la biologie pose problème à la méthodologie falsificationniste. Comme nous le disions, la théorie de l'évolution représente un cas problème pour son entreprise méthodologique. C'est un problème méthodologique qu'il faut éviter de confondre avec une condamnation on tologique de cette théorie. Darwin et les néo-darwiniens font d'une part de l'histoire naturelle en s'intéressant à un ensemble de données géographiques, géologiques, botaniques, morphologiques, paléontologiques, embryologiques et zoologiques qui leur servent à élaborer une explication historique. Ainsi, l'hypothèse évolutionniste (h1) explique ces données historiques multiples comme étant le résultat d'un processus d'engendrement des organismes à partir d'un ancêtre commun. Il ne fait aucun doute que ce qui intéresse alors l'historien naturaliste est de reconstruire l'histoire passée du processus évolutionnaire sur Terre dans toute sa singularité. Pour reprendre le modèle popperien de l'explication scientifique, l'hypothèse (h1) constitue l'explanans (ce qui explique) et les multiples données historiques (passées et présentes) constituent l'explanandum (ce qui est à expliquer).

Mais Darwin et les néo-darwiniens sont également des théoriciens lorsqu'ils cherchent à déterminer les « forces » et les mécanismes responsables de ce processus évolutif. Il s'agit alors d'expliquer comment a pu s'opérer l'engendrement des organismes à partir d'un ancêtre commun. Autrement dit, d'explanans, l'hypothèse (h1) devient un explanandum dont l'explanans est constitué des hypothèses (h2), (h3), (h4) et (h5). C'est pourquoi Popper introduit une division entre le sous-système ( $\mathrm{S} 1)$ qui correspond à l'hypothèse (h1) et le sous-système ( $\mathrm{S} 2)$ composé des quatre autres hypothèses.

Dans le présent exposé, nous tenterons de cerner le revirement popperien par rapport à 1 . la fonction, 2 . la testabilité, 3 . le statut logique et 4 . la valeur explicative de trois hypothèses : l'hypothèse évolutionniste (h1), l'hypothèse de la sélection naturelle (h4) et l'hypothèse de la survie des plus aptes (h5). 


\section{L'hypothèse évolutionniste (h1)}

Popper (1978: 344) considère donc maintenant que l'hypothèse de l'ascendance commune a été bien testée et on peut supposer par inférence (puisque Popper ne l'affirme pas explicitement) que c'est un énoncé testable, falsifiable, théorique et empirique. Cette inférence, dont nous examinerons maintenant les fondements, s'inscrit complètement à contre-courant de la première façon de voir de Popper. En effet, une quarantaine d'années auparavant, dans le premier de ses livres où on trouve une section consacrée au darwinisme, Misère de l'historicisme (1956) dont la première rédaction remonte en fait à $1935^{1}$, celui-ci prétend exactement le contraire, à savoir, que ce n'est pas un énoncé théorique et qu'en conséquence, il n'est ni empirique, ni falsifiable, ni testable. Insistons sur le fait que son questionnement est d'ordre exclusivement méthodologique. Il reconnaît en effet que l'hypothèse évolutionniste (h1) est "une brillante bypothèse scientifique relative a l'bistoire des divers espèces d'animaux et de végétaux sur la Terre» (1956: 108) et même qu'elle est "l'explication la plus pertinente desfaits en question." (1956: note 3 : 166). Il ne saurait donc être question, pour lui, de remettre en cause sa valeur scientifique ou de la condamner ontologiquement. N'empêche que cette hypothèse soulève des difficultés manifestes pour sa méthodologie falsificationniste du fait de son apparente ressemblance aux * lois * de l'histoire humaine formulées par les tenants de l' "historicisme", une philosophie des sciences sociales que Popper combat férocement à l'époque. C'est du fait de cette analogie entre l'explication darwinienne et les explications historicistes que Popper insistera exclusivement sur l'aspect historique de la théorie de l'évolution afin de montrer qu'elle ne se prête pas du tout au rôle justificateur que voudrait lui faire jouer l'historicisme en science sociale.

Lorsque l'hypothèse évolutionniste (h1) sert à expliquer un ensemble de faits particuliers amassés par des disciplines connexes sur l'évolution de la vie sur Terre, le néo-darwinien devient un historien et son hypothèse a le statut d'un énoncé d'existence singulier. En tant que telle, cette hypothèse n'est pas une "loi * de l'évolution mais bien la constatation de l'existence d'une tendance dans un processus unique. Lorsqu'on explique les données de la paléontologie en faisant appel à l'hypothèse de l'ascendance commune, on

1. Pour un excellent exposé de la petite histoire de la parution de Misère de Lhistoricisme, on se référera à R. Nadeau (1985: 3-5). 
ne les subsume pas sous une *loi " (en montrant que l'histoire de la vie sur Terre est un cas particulier de cette loi) mais on "décrit" un processus unique à l'aide d'énoncés singuliers. Le travail du biologiste est alors tout à fait similaire à celui de l'historien. Utilisée de cette façon donc, l'hypothèse évolutionniste (h1) est une assertion historique singulière : *Elle a, écrit Popper (1956: 108), le même statut que l'assertion bistorique: "Charles Darwin et Francis Galton avaient un grand-père commun". ".

Afin de ne pas accuser à tort Popper de confondre quoi que ce soit ici, insistons sur la différence entre l'explanandum d'une explication historique et l'explanandum d'une explication théorique. L'historien entend expliquer des événements situés dans le temps et dans l'espace qui se sont réellement déroulés. Dans le langage de The Logic of Scientific Discovery (1959: 62-4), l'énoncé explanandum d'une telle explication est un énoncé d'existence non strict. La mention de noms propres et de coordonnées spatio-temporelles est ici essentielle car sans cela on ne parlerait plus d'«histoire». C'est pourquoi, si le darwinien cesse de faire référence à des coordonnées spatio-temporelles, il cesse d'être historien et devient théoricien. Lorsqu'il fait abstraction des coordonnées spatio-temporelles de l'énoncé explanandum, d'énoncé existentiel non strict celui-ci devient un énoncé existentiel strict. Deux conséquences en découlent : 1. celui-ci ne parle plus d'événements réels mais d'abstractions et 2 . les événements dont il traite ne sont plus des événements singuliers dans toute leur spécificité mais bien des événements typiques et répétables. L'historien donc s'intéresse à des événements uniques alors que le théoricien s'intéresse à des événements répétables. Or, écrit Popper (1956: 109), "L'évolution de la vie sur Terre /.../ est un processus unique.».

S'il est possible de formuler les «lois* universelles à propos d'événements typiques et répétables, il n'est pas possible de le faire pour des événements ne survenant qu'une seule fois. C'est pourquoi le passé nous est inaccessible directement et ne nous est connue que par l'intermédiaire de monuments et de documents. Il n'est donc pas plus possible de formuler une loi inexorable de l'histoire humaine qu'il nous est possible d'en formuler une de l'évolution biologique. C'est cette impossibilité qu'entend mettre en évidence Popper à l'époque en qualifiant la croyance contraire d'"historicisme". L'historicisme est une théorie touchant les sciences sociales "qui fait de la prédiction 
bistorique leur principal but, et qui enseigne que ce but peut être atteint si l'on découvre les "rytbmes" ou les patterns, les *lois* ou les «tendances générales" qui soustendent les développements bistoriques. "(1956: 4). Si on pense que le biologiste peut prédire le cours futur de l'évolution de la vie sur Terre en s'appuyant sur sa connaissance du passé, nous introduisons certainement une forme de pensée pré-scientifique en biologie évolutionniste, à savoir, l'historicisme.

Du fait que l'évolution de la vie sur Terre est un processus unique, une hypothèse visant à l'expliquer ne peut pas être expérimentée directement et elle ne peut pas servir non plus à prédire l'occurrence d'un autre événement semblable. En fait, l'enjeu philosophique fondamental que voudrait défendre Popper contre l'historicisme est l'anti-déterminisme autant en ce qui concerne l'histoire humaine que l'histoire biologique. On ne peut prétendre extraire une finalité quelconque ni de l'une, ni de l'autre. Popper conçoit l'évolution de la vie sur Terre comme le fruit du "hasard* au sens de Cournot : « la rencontre fortuite de séries causales indépendantes":

Le point crucial est le suivant, écrit-il (1956: 117), bien que nous puissions admettre que toute succession réelle de phénomènes s'effectue en accord avec des lois de la nature, il est important de se rendre compte que pratiquement aucune séquence de supposons trois ou quatre événements concrets causalement reliés, ne s'effectue selon une quelconque loi unique de la nature.

Pour nous faire comprendre son idée, Popper fait appel à l'exemple célèbre de la pomme de Newton. Le vent souffle et une pomme tombe de l'arbre. Bien que la chute ellemême s'effectue selon les lois de la gravitation universelle, le vent qui souffle sur cet arbre particulier, à cet endroit et en ce temps particuliers est une rencontre fortuite de séries causales indépendantes. L'explication de cette chute fera appel à une conjonction de plusieurs lois mais il ne saurait y avoir une loi unique de la succession de ces événements. C'est pourquoi Popper (1956: 109) écrit à propos de l'évolution de la vie sur Terre :

Un tel processus, nous pouvons l'admettre, s'effectue en accord avec tous les genres de lois causales, par exemple, les lois de la mécanique, de la chimie, de l'hérédité et de la ségrégation, de la sélection naturelle, etc. On ne peut cependant pas le décrire comme une loi mais seulement comme une assertion historique singulière. 
Si l'hypothèse évolutionniste (h1) n'est pas une *loi * mais un énoncé existentiel, quelle peut bien être alors sa fonction? Cette hypothèse est un *point de vue historique". Comme on le sait, l'historien marxiste s'intéresse à la lutte des classes alors que l'historienne féministe s'intéresse à la lutte des sexes. Pour que leur explication respective de l'histoire humaine puisse être conséquente avec leur point de vue, il leur faudra privilégier certaines données historiques aux dépens d'autres moins directement pertinentes. Pour être autre chose qu'une simple énumération de faits disparates, une histoire doit opérer une sélection et, c'est là, de l'avis de Popper (1956: 148), la fonction d'une hypothès historique comme $(\mathrm{h} 1)$ :

$1 . . /$ il est indubitable qu'il ne peut y avoir d'histoire sans point de vue; comme les sciences naturelles, l'histoire doit être sélective si elle ne veut pas être étouffée sous une masse de pauvres matériaux et incohérente.

L'hypothèse évolutionniste (h1) permet d'organiser en un ensemble cohérent, tout en nous faisant comprendre un grand nombre de faits génétiques, paléontologiques, morphologiques, embryologiques, etc. Cependant, il importe de ne pas confondre cette grande valeur heuristique avec sa valeur théorique. Bien qu'elle nous fasse comprendre les phénomènes évolutifs, cette hypothèse ne permet pas de les prédire et, de ce fait, ne peut pas être testée directement. Comme le remarque Popper (1956: 149), "il y a nécessairement une pluralité d'interprétations fondamentalement équivalentes. ". On ne peut pas départager entre différentes interprétations de l'histoire sur la base de tests car le degré de testabilité de chacune d'entre elles est égal à zéro.

Afin de comprendre pourquoi l'hypothèse évolutionniste (h1) n'est pas « testable (au sens de Popper), rappelons ici quelques distinctions de The Logic of Scientific Discovery (1959) en nous servant de deux exemples d'énoncés tirés de l'*Introduction 1982 » à Realism and the Aim of Science (1983: xx). Une hypothèse est dite "testable" et "scientifique " si elle est falsifiable au moins en principe. Elle est falsifiable en principe si la classe de ses falsificateurs potentiels (par opposition à ceux actualisés) n'est pas vide. Un falsificateur potentiel est un énoncé de base décrivant un événement dont la possibilité est logiquement interdite par l'hypothèse. C'est pourquoi, si jamais cet événement se produit, on dira que l'hypothèse a été "contredite par les faits". Considérons les exemples suivants :

(E1) "Tous les cygnes sont blancs.» 
(E2) «Il existe une couleur qui provoque la terreur chez ceux qui la regardent."

L'énoncé (E1) est falsifiable en principe car il peut logiquement être contredit par l'énoncé existentiel non strict suivant.

(E3) «Le 16 mai 1934 entre 10 et 11 heures, il y avait un cygne noir devant la statue de l'impératrice Elizabeth à Vienne.".

Mais, pour pouvoir falsifier une hypothèse, un événement ne doit pas être simplement hasardeux (comme un effet non reproductible survenu au cours d'une seule expérimentation) mais répétable. C'est pourquoi, nous exigerons du falsificateur potentiel qu'il puisse être érigé en un énoncé existentiel strict (dont on aura fait abstraction des coordon nées spatio-temporelles) :

(E4) «Il existe au moins un cygne noir".

Logiquement, (E4) contredit (E1) de telle sorte que si nous acceptons de considérer le premier comme décrivant un état de fait, alors (E1) sera réfuté par les faits.

Au contraire, l'énoncé (E2) est logiquement infalsifiable, c'est-à-dire que la classe de ses falsificateurs potentiels est vide. En raison de sa nature logique d'énoncé existentiel, on peut "vérifier" qu'il existe une couleur provoquant la terreur si le phénomène se déroule sous nos yeux mais nous serions incapables de spécifier un événement pouvant le contredire. En effet, l'énoncé :

(E5) *Il existe une couleur ne provoquant pas la terreur* multiplié par autant de couleurs que nous pourrons observer $\left(E_{6} \ldots E_{n}\right)$ ne pourra jamais contredire logiquement (E2). Le seul énoncé pouvant le contredire est l'énoncé :

(E6) *Aucune couleur ne provoque la terreur chez ceux qui la regardent *

qui a un caractère universel. Seul un énoncé universel peut contredire un énoncé existentiel mais ce genre d'énoncé n'est pas admissible en tant que falsificateur potentiel car ce n'est pas un énoncé de base, c'est-à-dire qu'il n'est pas "vérifiable".

Même si l'hypothèse évolutionniste n'est pas falsifiable et n'est pas testable, cela ne signifie pas qu'on ne puisse pas la "confirmer" ou la "vérifier" à partir des multiples données de la morphologie, de l'embryologie ou de la paléon- 
tologie. Mais, aux yeux de Popper, ce n'est pas là la façon scientifique de procéder. En effet, le marxiste convaincu trouvera des * confirmations * de la lutte des classes à chaque page de son journal quotidien y compris les annonces publicitaires. Il serait difficile de prétendre qu'il s'agit là d'un test "scientifique" de ces hypothèses.

L'hypothèse évolutionniste (h1) ne peut pas servir à justifier l'historicisme en science sociale car elle $n$ 'introduit aucun «déterminisme» dans l'évolution de la vie sur Terre et en conséquence, ne peut pas être formulée comme une "loi * unique d'une séquence d'événements mais seulement comme un énoncé existentiel décrivant cette séquence. Tel est donc le message que semble vouloir nous livrer Popper qui combat à l'époque les conceptions «déterministes» de l'histoire humaine. Au terme de cette analyse, nous savons maintenant que la première incursion de Popper en philosophie de la biologie, celle qui nous est livrée dans Misère de l'historicisme (1956) concerne en fait un usage "parascientifique» de l'hypothèse évolutionniste (h1) qu'il examine prise isolément en tant qu'explanans pour une série de données recueillies par plusieurs disciplines comme la paléontologie, l'embryologie comparée, la systématique zoologique, etc. Cette perspective s'explique du fait qu'il s'agit de comparer la prétendue "loi de l'évolution biologique" avec les prétendues "lois de l'évolution de l'histoire humaine" des historicistes. Ainsi considérée, il s'agit d'une hypothèse historique, d'une interprétation de l'histoire naturelle. On ne peut certes prétendre expérimenter une telle interprétation à la manière d'une loi de la génétique. Son statut logique est celui d'un énoncé existentiel non strict et en tant que tel, elle n'est ni falsifiable, ni testable logiquement. Sa fonction est de servir de principe organisateur nous permettant de comprendre de nombreux faits.

\section{L'hypothèse évolutionniste (h1) est-elle testable?}

Comme nous le savons, en 1977, Popper semble changer d'idée sur la testabilité de cette hypothèse puisqu'il affirme qu'elle a été bien testée. Malheureusement, Popper ne nous en dit pas plus long dans son article de 1978. Le lecteur est donc livré à lui-même pour reconstruire le reste de son argument. La reconstruction la plus naturelle consiste à supposer que Popper n'avait nul besoin d'expliciter plus amplement son revirement car, on sait qu'il établit 
une équivalence logique entre la "testabilité *, la *falsifiabilité ", la * théoricité \# et l'*empiricité * d'une théorie : si (h1) a été bien testée (comme il le dit explicitement), c'est qu'elle est testable et si elle est testable, alors elle est falsifiable, théorique et empirique (ce que Popper ne dit pas explicitement). La difficulté soulevée par cette interprétation est de devoir changer le statut logique de l'hypothèse évolutionniste (h1). En effet, la falsifiabilité est une relation logique entre un énoncé strictement universel et un énoncé existentiel strict qu'il ne faut pas confondre avec la fausseté d'un énoncé. Même si un énoncé est rendu faux, cela ne change en rien son caractère falsifiable. De plus, un énoncé faux (un énoncé existentiel, par exemple) n'est pas pour cela, falsifiable. La falsifiabilité est la possibilité logique d'être contredit par un énoncé existentiel strict. Or, comme nous le disions, un énoncé existentiel strict ne peut pas contredire un autre énoncé existentiel strict ou non strict. La seule façon est de nier l'énoncé existentiel, ce qui équivaut logiquement à formuler un énoncé strictement universel. Pour être falsifiable donc, l'hypothèse évolutionniste (h1) ne doit plus être considérée comme un point de vue historique, un énoncé existentiel non strict, mais comme un énoncé strictement universel. Mais en ce cas, il serait possible, comme le suppose les historicistes, de formuler une loi universelle de l'évolution biologique et, de ce fait, d'en formuler une de l'histoire humaine. Autrement dit, changer le statut logique de l'hypothèse évolutionniste (h1) conduit à une forme de déterminisme historique et à la possibilité d'une science de l'histoire, des idées que Popper n'a jamais cessé de combattre toute sa vie. Contrairement à l'interprétation spontanée du revirement popperien, un revirement d'une telle envergure aurait certainement gagné à être explicité davantage.

Il semble plus plausible de penser qu'en opérant ce revirement dans un texte aussi court, Popper ne pensait pas remettre en cause une partie aussi importante de sa pensée. Autrement dit, le retournement popperien ne concerne pas l'hypothèse évolutionniste dans le contexte de Misère de l'historicisme (1956) mais plutôt, un autre aspect de sa pensée biologique qu'il développera plus tard. Mais avant de considérer les autres étapes dans le développement de la pensée popperienne, examinons les fondements logiques d'une telle interprétation de son revirement. Le problème se pose de la façon suivante : Popper peut-il de façon cohérente avec sa méthodologie, considérer que l'hypothèse (h1) a été testée sans changer son statut logique (un énoncé 
existentiel non strict), sa fonction (un guide dans la recherche d'explication), son degré de testabilité (égal à zéro) et sa valeur explicative (un principe organisateur nous permettant de comprendre mais sans pouvoir prédire)? Pour répondre à cette question, traçons une distinction entre *être logiquement falsifiable " et *être méthodologiquement falsifiable». Ainsi que nous l'avons dit un énoncé existentiel comme (h1) n'est pas logiquement falsifiable mais n'empêche qu'il peut le devenir méthodologiquement. Si prise isolément, la classe de ses falsificateurs potentiels est vide, la classe des falsificateurs potentiels de sa conjonction avec des énoncés universels n'est pas vide. C'est pourquoi, si nous pouvons corroborer les énoncés universels d'une façon indépendante, en cas de réfutation de la conjonction, nous serons justifiés de prendre la décision méthodologique d'en tenir l'énoncé existentiel responsable. Considérons l'exemple suivant :

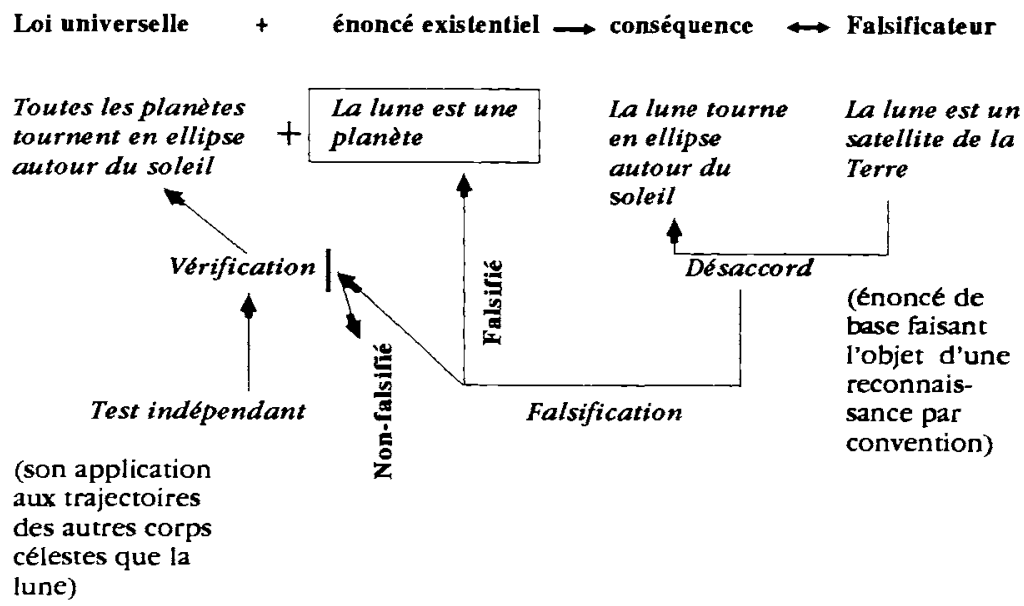

Dans ce schéma, un énoncé existentiel logiquement infalsifiable ( $*$ La lune est une planète $*$ ) est mis en conjonction avec un énoncé universel (*Toutes les planètes tournent en ellipse autour du soleil $*$ ) et de cette conjonction, est déduite une conséquence logique («la lune tourne en ellipse autour du soleil $»$ ) qui est contredite par un falsificateur potentiel ("La lune tourne en ellipse autour du soleil») qui est contredite par un falsificateur potentiel («Ia lune tourne en ellipse autour de la Terre $*$ ). Si nous accordons notre confiance à la loi de Kepler pour l'avoir testée d'une façon indépendante, nous serons justifiés méthodologique- 
ment d'attribuer la responsabilité de la réfutation à l'énoncé existentiel. Ainsi, un énoncé existentiel qui pris isolément est logiquement infalsifiable, en conjonction avec un énoncé universel, peut devenir méthodologiquement falsifiable.

Autrement dit, prise isolément (dans le contexte de Misère de l'historicisme), l'hypothèse évolutionniste (h 1) n'est pas testable mais en conjonction avec des énoncés universaux (le contexte d'après 1956), elle peut devenir méthodologiquement falsifiable. Pour répondre à la question concernant les fondements logiques de la présente reconstruction de son revirement de 1977 : oui, Popper peut très bien continuer à considérer l'hypothèse évolutionniste (h1) comme un énoncé existentiel non testable, non falsifiable et non prédictif tout en soutenant de façon conforme aux canons de sa méthodologie qu'elle a bien été testée. Popper ne se rétracte pas réellement par rapport à la valeur scientifique de cette hypothèse. Cette idée suppose que Popper voulait remettre en question la valeur scientifique de cette hypothèse et c'est là mal comprendre ses intentions. Dans Misère de l'historicisme (1956), Popper ne veut pas montrer que l'hypothèse évolutionniste n'est pas «scientifique ", n'est pas «empirique* et ne peut pas être "testée * dans son usage scientifique (c'est-à-dire en conjonction avec des énoncés universaux). Ce n'est pas la connaissance biologique que Popper veut remettre en question mais bien la conception que se font les historicistes de cette connaissance. Ce que veut montrer Popper est que l'hypothèse évolutionniste ne peut pas servir d'appui à l'historicisme dans les sciences sociales. Les historicistes de l'époque, semble-t-il, entendaient comparer leurs lois de l'évolution de l'histoire humaine à l'hypothèse évolutionniste dans le but de leur conférer une certaine crédibilité. Comme on le sait, Marx et Engels se réclameront explicitement de Darwin. Mais comme le montre Popper, cette comparaison est fondée sur une mauvaise compréhension de cette hypothèse par les historicistes mais non pas par les biologistes euxmêmes. Popper nous invite donc à distinguer entre un usage scientifique de (h1) qui est historique et non déterministe et un usage pseudo-scientifique ou para-scientifique qui est prophétique et déterministe. S'il n'y a pas de loi (unique) de l'évolution biologique, à plus forte raison, il ne saurait y en avoir de l'histoire humaine. C'est là une contribution durable de Popper aux débats philosophiques entourant la théorie de l'évolution qui est facilement mal interprétée si on croit erronément qu'il attaque la conception que se font les biologistes de cette hypothèse plutôt que celle 
que s'en font les historicistes. En considérant que l'hypothèse (h1) a été bien testée, Popper n'a pas changé d'idée mais simplement de perspective. Avant, il questionnait un usage para-scientifique de cette hypothèse alors qu'après il parlera de son usage scientifique.

3. Le sous-système (S2) des hypothèses (h2), (h3), (h4) et (h5)

À partir de son Postcript After Twenty Years écrit au cours des années 50 mais qui n'a été publié que tout récemment (1982-3), Popper se tourne vers l'usage scientifique de cette hypothèse et sa conjonction aux autres hypothèses du système théorique néo-darwinien. Bien qu'il continue à utiliser les mêmes noms et les mêmes dénominations (comme "théorie de l'évolution *, "darwinisme*, "théorie néo-darwinienne», etc.), il fait référence cette fois au système des hypothèses explicatives de (h1). Puisque Popper ne discute pas de (h2) (les aspects génétiques) et qu'il examine (h3) uniquement sur le plan scientifique (comme nous le disions au début en parlant des différents niveaux de son intervention en biologie évolutionniste), son objet est maintenant la conjonction de (h1), (h4) et (h5).

Dans The Open Universe (1982a), Quantum Theory and the Scbism in Physics (1982b) et Realism and the Aim of Science (1983), Popper considère que le grand mérite scientifique du système explicatif néo-darwinien est d'avoir montré comment on peut remplacer l'ancienne explication téléologique et finaliste de l'apparition des diverses formes de vie sur Terre par une explication causale formulée dans des termes physicalistes et faisant intervenir un mécanisme naturel, à savoir, la sélection naturelle. Mais attention, car la nuance est importante : à son avis, le système théorique néo-darwinien ne nous fournit pas une telle explication. Il nous indique simplement dans quel sens chercher une telle explication. Il montre simplement qu'une telle explication est possible. En fait, Popper rapproche la théorie néo-darwinienne d'autres théories scientifiques telles la théorie atomique d'avant le $\mathrm{XX}^{\mathrm{e}}$ siècle, la théorie des champs de forces de Faraday et Maxwell ou la théorie des champs unifiés d'Einstein qui, tout en n'étant pas testables, ont néanmoins guidé et favorisé le progrès de la connaissance scientifique. À ce genre de théorie, Popper donne le nom de "programme métaphysique de recherche*. 
Chacune de ces théories métaphysiques, écrit-il (1983: 192$3)$, avant de devenir testables, a servi de programme de recherche pour la science. Elles ont indiqué une direction de recherche et le genre d'explication pouvant nous satisfaire; de plus, elles ont rendu possible quelque chose comme une appréciation en profondeur des théories. En biologie, la théorie de l'évolution, la théorie cellulaire et la théorie de l'infection bactérienne ont joué un rôle similaire, au moins pendant un certain temps.

Par " théorie de l'évolution ", Popper entend la conjonction de (h1), (h2), (h3), (h4) et (h5). Il spécifie clairement ici qu'elles ont été conjointes "pendant un certain temps". Ainsi, l'explication originale de Darwin sans les fondements génétiques néo-darwiniens était nettement un programme métaphysique de recherche au sens où l'entend Popper. Ce genre de théorie se caractérise par trois traits distinctifs : 1 . une telle théorie a un caractère programmatique, c'est-àdire qu'elle ne constitue pas une véritable solution au problème d'explication mais une indication dans quel sens trouver la solution :

/.../ ces idées, écrit Popper (1982b), ne déterminent pas seulement à quels problèmes nous choisirons de nous attaquer, mais également quel type de réponse nous satisfait et ce que nous considérons comme une amélioration ou un progrès par rapport aux réponses antérieures.

2. elle se distingue également par son incapacité à être testée; 3. enfin, il y a toujours la possibilité d'autres programmes de recherche tout aussi justifiables. Dans The Open Universe (1982a : 150), Popper précise que cette dernière caractéristique ne s'applique pas dans le cas du programme néo-darwinien: «Pour le moment, il n'y a pas d'autres bypotbèses compétitives sérieuses.».

Mais, c'est véritablement dans Objective Knowledge (1972) que Popper explicite sa façon de caractériser le système théorique néo-darwinien et plus spécifiquement la conjonction de (h1), (h4) et (h5). C'est d'ailleurs à ce livre qu'il renvoie le lecteur désireux d'approfondir sa pensée d'avant sa rétractation dans son article de 1978 (note 7 : 344). A la suite de plusieurs spécialistes de la biologie évolutive, Popper craint que l'explication néo-darwinienne ait un caractère circulaire.

/.../ la difficulté à propos de la théorie de l'évolution, écrit-il (1972: 241), est son caractère tautologique ou quasi-tautologique. 
La terminologie inconséquente de Popper saute ici aux yeux. Dans Misère de l'historicisme (1956), cette expression référait à l'hypothèse évolutionniste (h1) prise isolément comme nous l'avons vu. Or, il est évident qu'un énoncé existentiel non strict affirmant l'occurrence d'un événement non répétable comme l'ascendance commune des formes de vie sur Terre, ne saurait être caractérisé comme un énoncé tau tologique ou une explication circulaire. Cette expression fait désormais référence à la conjonction de (h1), (h4) et (h5) bien qu'il soit toujours sous-entendu que le système complet comprend également (h2) et (h3).

À son avis donc, la chaîne des définitions des concepts centraux de ce système théorique tels que les concepts d' "aptitude*, d' «adaptation *, de "survie du plus apte " et de « sélection " conduit à une certaine circularité dans l'explication, non pas logiquement mais circonstanciellement. La distinction est importante car on ne saurait accuser Popper de confondre ici une définition théorique et une définition opératoire. Popper ne dit pas que ces définitions sont « tautologiques" mais bien qu'elles sont "quasi-tautologiques". Selon l'explication néo-darwinienne, les descendants reproduisent assez fidèlement les traits parentaux (selon h 2) et, dans le processus de reproduction, de petites modifications apparaissent fortuitement causant des mutations (selon h3) que la sélection naturelle élimine (selon h4) lorsqu'elles sont mal adaptées à l'environnement, favoriasnt ainsi la reproduction des plus aptes (selon h5). Dans la lignée héréditaire, il se produit au hasard de petits changements dans l'acide nucléique des gènes causant l'apparition de caractères différents de ceux des parents. Ces mutations sont ensuite triées par la sélection naturelle : la lignée héréditaire qui hérite d'une mutation favorable s'adapte mieux à l'environnement et peut ainsi survivre plus longtemps et se reproduire à un taux plus élevé.

Comme l'ont noté plusieurs avant Popper, la difficulté de cette explication est qu'on estime l'adaptation d'une population ou d'une espèce à son taux de reproduction ou à son taux de survie. Popper se restreint à parler uniquement du taux de survie. Si on prétend ensuite expliquer ce taux de survie en termes d'adaptation à l'environnement, nous nous engageons dans une circularité certaine. En effet, si nous définissons l'adaptation par la survie, il est trivial de considérer après coup qu'une population ayant survécu est une population adaptée à son environnement. Il est tout aussi trivial d'affirmer qu'une espèce ou une population en 
voie de disparition est mal adaptée à son environnement. "Être plus apte " signifie ici la même chose qu' " avoir survécu *.

Afin d'éviter qu'on accuse à tort Popper de confondre des définitions théorique et opératoire, insistons sur la différence entre une * tautologie » et une * quasi-tautologie *. Considérons les deux énoncés suivants :

(E10) «Ceux qui survivent sont les plus aptes», et

(E11) "Ceux qui survivent sont ceux qui survivent".

Si (E11) est logiquement une tautologie, (E10) par contre n'en est pas une. Cela dépend de la façon dont nous définissons «être plus apte*. Si on ne peut pas le faire opérationnellement autrement qu'en termes de survie, alors (E10) est transformé en un énoncé aussi tautologique que (E11).

/../ nous n'avons pas, j'en ai bien peur, écrit Popper (1972: 242), d'autre critère de l'adaptation que la survie actuelle et, c'est pourquoi, nous concluons que les organismes les plus aptes et les mieux adaptés à leurs conditions de vie sont ceux qui ont survécus.

Autrement dit, si notre interprétation de l'explication néo-darwinienne conduit à devoir affirmer que les organismes mal adaptés périssent nécessairement, alors il est tout à fait circulaire d'en conclure que ceux qui ont survécus sont nécessairement bien adaptés.

Cette formulation, écrit-il (1972: 69), est presque tautologique parce que * pour le moment bien adapté * veut dire à peu près la même chose qu' «avoir les qualités qui l'ont fait survivre jusqu'ici». En d'autres mots, une partie considérable du darwinisme n'a pas la nature d'une théorie empirique mais bien celle d'un truisme logique.

Il est clair que Popper discute ici uniquement de la conjonction de (h4) et (h5) qui, comme nous le savons, explique ( $\mathrm{h} 1)$. Si on pose une connexion nécessaire entre "aptitude *, «adaptation * et *survie», la conjonction de (h4) et (h5) devient parfaitement tautologique. Il va de soi qu'aucun cas de disparition ou de survie d'un organe, d'une population ou d'une espèce ne saurait contredire cette connexion nécessaire entre "aptitude", "adaptation" et "survie" car les trois sont référentiellement équivalents. C'est pourquoi, une telle explication n'est pas «testable» au sens de Popper.

Considérons maintenant la fonction et la valeur explicative de ce programme métaphysique (parce que non testa- 
ble) de recherche. F.A. von Hayek (1967) considère important de noter que le degré de complexité des phénomènes étudiés dans les sciences de la nature et dans les sciences sociales n'est pas le même. Les phénomènes sociaux comprennent toujours un grand nombre de facteurs capables d'agir significativement dans une situation alors que les phénomènes physiques peuvent s'expliquer par un petit nombre d'entre eux (la majorité des autres facteurs n'étant pas pertinents). Pensons, par exemple, à la chute libre d'un corps dans un arrangement expérimental très bien contrôlé en comparaison à un système économique comprenant 40 millions d'individus et un nombre encore plus grand de marchandises. Dans ces conditions, selon Hayek, il est clair que nous ne parviendrons jamais à expliquer les réactions d'un tel système économique d'une façon aussi précise que la chute libre d'un corps. Non pas qu'on ne puisse pas l'expliquer mais tout simplement que l'explication d'un phénomène social n'ira jamais dans un degré de détail équivalent. Les explications en sciences sociales resteront toujours un peu "floues». Pour bien marquer cette différence et éviter le «scientisme* (c'est-à-dire l'imitation servile de la méthode des sciences de la nature en sciences sociales), Hayek propose de distinguer entre des explications du détail et des explications du seul principe des phénomènes. Dans la quatrième section du chapitre 1 de Studies in Pbilosophy, Politics and Economics, Hayek avance l'idée qu'en raison de la complexité des phénomènes évolutifs, la *théorie de l'évolution * ne peut véritablement offrir une explication que du seul principe et non du détail des phénomènes évolutifs. Le genre d'explication propre à la physique lui est inaccessible. Autrement dit, l'explication néo-darwinienne nous fait comprendre le principe des évolutions et des adaptations mais la connaissance de ce principe ne saurait nous permettre de prédire ensuite l'occurrence de tel événement particulier dans un temps et dans un lieu déterminés. Tout ce qu'on peut dire est qu'un certain événement typique pourrait survenir dans une situation typique. Popper (1972: 267) semble entièrement se rallier à cette idée lorsqu'il écrit :

Bien que ce soit une grande réussite, nous devons ajouter que l'expression "en principe» désigne une restriction importante. Ni Darwin, ni aucun darwinien n'a jusqu'ici fournit une explication causale de l'évolution adaptative réelle d'un seul organe ou d'un seul organisme. Tout ce qui a été montré et c'est beaucoup - est que de telles explications pourraient exister (c'est-à-dire qu'elles sont logiquement possibles) 
Même si une telle explication paraît possible *en principe », concrètement, le nombre de facteurs impliqués dans une situation réelle d'adaptation est beaucoup trop grand pour pouvoir en donner une explication causale particulière. Â la suite de Popper, distinguons entre un système naturellement isolé, tel le système solaire, et un système artificiellement isolé par la pensée (ou même concrètement comme des cages à population de drosophiles ou des petits écosystèmes de coyotes et de lapins). Dans les deux cas, l'astronome et le biologiste travaillent sur des "modèles * construits de situation réelle - un modèle ne pouvant par principe représenter qu'une partie de la situation réelle. Néanmoins, le modèle de l'astronome parvient à isoler les seuls propriétés pouvant agir dans la situation étudiée alors que celui du biologiste isole artificiellement certaines propriétés en faisant abstraction d'autres qui pourraient également influencer cette situation. Le biologiste travaille sur des modèles extrêmement simplifiés et c'est pourquoi, il ne peut expliquer que le seul principe de situation typique simplifiée.

La théorie de la sélection naturelle, écrit Popper (1972 : 269), procède de façon similaire. Dans l'explication d'un cas particulier, elle commence avec un modèle simplifié de la situation - une situation constituée de certaines espèces dans certaines conditions expérimentales - et tente de montrer pourquoi, dans cette situation, certaines mutations auraient une valeur de survie.

Pour bien marquer la différence entre l'explication de l'astronome et celle du biologiste, Popper in troduit le terme de *simulation*: l'explication néo-darwinienne nous fait comprendre le principe de certaines situations typiques en les simulant plutôt qu'en les expliquant. Pour expliciter sa pensée, Popper avance une analogie. Supposons un modèle simplifié de la situation typique d'une éclipse lunaire constitué d'une lampe et de deux petits bloc de bois. La simulation de la situation réelle consiste à faire tourner le premier bloc de bois autour de la lampe et le second autour du premier simultanément. Nous serons à même de nous représenter et de comprendre ainsi le principe des éclipses lunaires mais nous serions incapable de prédire, à l'aide de ce modèle, l'occurrence de la prochaine éclipse lunaire.

Dans la section intitulée *Le darwinisme comme programme métaphysique de recherche * de son *Autobiography, Popper reprend sensiblement les mêmes thèses qu'il a développées dans Objective Knowledge. 
J'en suis venu à la conclusion, écrit-1l (1974: 134), que le darwinisme n'est pas une théorie scientifique testable, mais un programme métaphysique de recherche - un cadre théorique possible pour des théories scientifiques testables.

Popper insiste à nouveau sur le caractère *quasi tautologique * de cette explication qui la rend non testable. Par exemple, sur la base de cette explication peut-on affirmer que sur une autre planète (Mars) où on trouverait des formes de vie respectant les conditions spécifiées par les hypothèses de l'hérédité (h2), de la mutation au hasard (h3) et de la sélection naturelle (h4), il y aurait nécessairement une grande variété de formes distinctes comme sur la Terre? Non, selon Popper, car supposons que nous y découvrions seulement trois espèces de bactéries ayant un code génétique similaire aux espèces terrestres. L'explication néo-darwinienne de la vie sur Terre ne serait jamais remise en question pour autant puisque, selon l'hypothèse (h5), nous pourrions dire que c'étaient là les seules espèces à pouvoir survivre sur cette planète. Autrement dit, */.../ le darwinisme, écrit Popper (1974: 136), ne prédit pas vraiment l'évolution des variétés. En conséquence, il ne peut pas non plus l'expliquer.". Tout ce qu'on peut prédire est que dans certaines circonstances favorables, il y aura une grande variété de formes. Mais, on demeure incapable de préciser davantage ce qu'il faut entendre par l'expression "dans certaines circonstances favorables $*$.

Dire qu'une espèce vivante, écrit Popper (1974: 137), est adaptée à son environnement est, en fait, presque une tautologie. En effet, nous utilisons les termes *adaptation * et «sélection" de façon telle que nous sommes conduits à dire qu'une espèce non adaptée aurait été éliminée par la sélection naturelle.

La seule prédiction permise par le système théorique néo-darwinien qui ne soit pas un truisme logique est celle voulant que cette évolution se fasse de façon graduelle. Mais, là encore, l'explication nous fait comprendre le seul principe de l'évolution graduelle tout en ne nous premettant pas de prédire l'occurrence de petits changements particuliers. Malgré cette lacune, la théorie de l'évolution nous invite à toujours chercher des étapes intermédiaires entre les changements importants et c'est pourquoi sa fonction est celle d'un programme métaphysique de recherche. Comme on peut le constater, c'est vraiment la conjonction de (h1), (h4) et (h5) qui pose problème à Popper. 


\section{Le grand retournement}

Ainsi que nous l'avons annoncé, Popper (1978: 345) change d'idée au sujet de sa caractérisation de la conjonction de (h1), (h4) et (h5) :

Je crois encore que la sélection naturelle fonctionne de cette façon comme un programme de recherche. Néanmoins, j'ai changé d'idée à propos de la testabilité et du statut logique de la théorie de la sélection naturelle; et je suis content d'avoir l'occasion de faire cette rétractation.

L'expression «théorie de la sélection naturelle * fait ici référence à la conjonction de (h4) et (h5). Afin de bien comprendre le sens de cette rétractation, spécifions plus précisément en quoi consistait l'ancienne conception popperienne. La première chose a rappelé est certainement que Popper est en bonne compagnie pour souligner ce qu'on appelle le «tautology problem " de la théorie de la sélection naturelle. Popper avoue avoir été influencé par les plus grands théoriciens de cette discipline. Il y avait donc bien là une difficulté réelle de la théorie et Popper n'était pas seul à le penser.

Il est également important de distinguer, comme Sober (1984a), les deux aspects du problème de la tautologie: certains posent une relation de synonymie en tre "aptitude *, *adaptation * et *survie* de telle sorte que le problème est celui d'une définition théorique a priori. D'autres, comme Popper, sont surtout concernés par la définition opératoire de ces termes. Pour ces derniers, le problème

/... est qu'il n'y a aucun moyen, écrit Sober (1984a: 80), de découvrir comment un organisme est apte à survivre autrement qu'en observant comment il réussit avec succès à survivre et à se reproduire. Nos jugements à propos de l'aptitude à s'adapter sont en conséquence ex post facto plutôt que prédictifs.

Autrement dit, il n'y a pas d'autre critère indépendant que la survie actuelle et le taux de reproduction actuelle pour déterminer opérationnellement l'aptitude à s'adapter d'un organisme. À la suite de Gould (1977) qui, en réponse à cette objection, propose des critères indépendants (des caractéristiques morphologiques, comportementales ou physiologiques), Alexander Rosenberg (1980) et (1985) pense que le taux de reproduction passé n'épuise pas la signification empirique du terme "aptitude à s'adapter *. En effet, s'il en était autrement alors un organisme 01 artificiellement créé en laboratoire et ayant exactement la même constitution anatomique et physiologique qu'un organisme 
naturel 02, ne serait pas considéré comme ayant les mêmes chances de survie dans un même environnement. Et pourtant, l'identité des propriétés physiologiques et anatomiques nous fera affirmer le contraire, à savoir, que leur aptitude à s'adapter et à survivre dans le même environnement est à peu près équivalente. De même, l'écureuil et l'oiseau ont des constitutions physiologiques et anatomiques fort différentes mais ils peuvent tous deux s'adapter à un même environnement physique. Autrement dit, en plus de son aspect reproductif (taux de reproduction), la notion d'aptitude à s'adapter a également des aspects physiologique, anatomique et environnemental (botanique, géographique, etc.). Ces aspects font également partie de la signification empirique du terme "aptitude à s'adapter" et peuvent servir à déterminer de façon indépendante les capacités de survie d'un organisme dans un environnement. Cependant, il est clair que la diversité des facteurs impliqués par ces aspects ne permet pas de les subsumer sous une définition générale et c'est pourquoi nous n'avons d'autre choix que de définir cette aptitude de l'organisme à s'adapter en termes de taux de reproduction passé. L'important est que l'explication n'est pas circulaire.

En fait, la définition que donne Darwin de l'expression "aptitude à s'adapter" comporte deux composantes : 1 . la capacité de survie jusqu'à l'âge de reproduction et 2 . la capacité de se reproduire. Si Sober, Gould et Rosenberg insistent sur la seconde, Popper lui insiste sur la première. On ne peut pas déterminer si un organisme est apte à s'adapter autrement qu'en observant après coup qu'il a survécu dans un certain environnement. De plus, le problème dont parle Popper n'est pas un problème linguistique (la signification empirique d'un terme) mais un problème méthodologique, à savoir, si ces termes insérés dans une explication permettent de prédire et de tester la théorie. Pour être testable et explicative, la théorie doit permettre de prédire la survie et non simplement de la constater après coup. Si nous avons l'illusion que cette théorie explique quelque chose, c'est que nous savons déjà que l'organisme a survécu mais nous serions incapable de le prédire. Même l'exemple contraire de Rosenberg ne le fera pas. En effet, si nous prédisons que l'organisme artificiellement créé (ou un autre organisme futur et similaire, pour reprendre la solution de Sober 1984a) survivra dans un certain environnement, c'est parce qu'il ressemble à l'organisme dont nous savons déjà qu'il a survécu dans cet environnement. C'est cette incapacité à prédire véritablement qui gêne Popper car, sans cette possibilité, on ne peut pas tester l'explication d'une façon indépendante 
de l'explanandum pour laquelle elle a justement été conçue. Nous ne faisons alors que "re-confirmer" sans cesse l'explication avec le même phénomène. Cette façon de faire ne peut constituer un test sérieux car nous ressemblons alors au marxiste convaincu qui trouve dans son journal quotidien des "confirmations" de la théorie du Matérialisme historique.

Distinguons ici deux raisons pour lesquelles Popper pense que la théorie de la sélection naturelle ne peut pas être testée: 1. de par son statut logique de "quasi-tautologie " ne permettant qu'une explication ex post facto et 2 . du fait que c'est une explication du seul principe des phénomènes ne permettant pas assez de précision pour être véritablement confrontée à l'expérience. Laissons de côté la seconde raison pour le moment car la rétractation popperienne porte uniquement sur la testabilité et le statut logique de la théorie de la sélection naturelle.

Pour justifier sa rétractation, Popper avoue, dans son texte de 1978, avoir confondu la très grande difficulté de tester cette théorie avec l'impossibilité de la tester. Popper n'a jamais contesté que cette théorie avait une signification empirique car, comme il le dit, dans certains cas, on peut presque voir la sélection naturelle opérer sous nos yeux. Ce que conteste Popper est que ces "confirmations" puissent servir de « tests * à la théorie. Ces confirmations ressemblent plus à des applications du programme de recherche. Or, ce que présuppose logiquement ces applications et ce programme de recherche est un principe universel selon lequel «seules les aptitudes à s'adapter favorables permettent de survivre, les autres étant éliminées". Ce qui gêne Popper est la connexion nécessaire établie entre "aptitude ", "adaptation" et "survie». Les aptitudes des organismes ayant survécu sont nécessairement favorables à leur adaptation et celles des organismes en voie de disparition sont nécessairement défavorables à leur adaptation. Mais, même en continuant de toujours chercher à expliquer les phénomènes évolutifs de cette façon, par décision méthodologique, le biologiste n'est pas tenu de croire en l'universalité de ce principe. En admettant la possibilité de certaines mutations défavorables ne menant pas nécessairement à leur élimination, le biologiste admet que son explication pourrait être contredite par les faits et être ainsi rendue falsifiable et testable. Mais, comme les données de diverses sciences connexes montrent que la survie de mutations défavorables est l'exception plutôt que la règle, on peut dire que la théorie a été bien testée. Clairement, une explication pouvant être contredite 
par les faits dans certains cas, n'est pas une tautologie. C'est pourquoi Popper (1978: 346) écrit :

Il semble préférable d'admettre que ce n'est pas tout ce qui évolue qui est utile, même si c'est étonnant de constater combien de choses le sont /.../En d'autres mots, il semble que, comme de nombreuses théories en biologie, l'évolution par la sélection naturelle n'est pas strictement universelle, même si elle semble tenir bon dans un grand nombre de cas importants.

En faisant de la théorie de la sélection naturelle un énoncé probabiliste (son nouveau statut), nous la rendons non seulement testable mais testée. Nous touchons donc ici au cour de la rétractation popperienne. Il croyait, semblet-il, auparavant qu'on ne pouvait admettre la possibilité de mutation défavorable non éliminée alors que maintenant, il croit cela possible. Si nous admettons la possibilité que de telles mutations défavorables persistent, c'est que l'aptitude à s'adapter peut être définie indépendamment de la survie actuelle et c'est ce qui importe à Popper. Évidemment, cela demeure toujours très difficile de tester la théorie du fait de sa valeur explicative (peu prédictive) mais au moins il n'y a plus d'objection de principe à de tels tests. Cependant comme sa fonction et sa valeur explicative ne changent pas, elle demeure largement une explication ex post facto.

\section{Conclusion}

Tout au long de cet exposé, nous avons voulu reconstruire la position de Popper en biologie évolutionniste de façon à montrer qu'elle est loin d'être limpide et univoque comme le supposent la plupart de ses critiques. Nous avons été conduit à distinguer trois périodes différentes dans l'élaboration de sa position (bien que nous n'ayons aucune prétention à l'avoir montré historiquement) : 1) la période de Misère de l'historicisme (1956) au cours de laquelle Popper examine l'hypothèse évolutionniste (h1) prise isolément en tant qu'explanans pour une multitude de faits historiques ; il la considère comme une hypothèse historique singulière dont le statut logique est celui d'un énoncé existentiel non strict et qui, de ce fait, n'est ni falsifiable, ni testable; sa fonction et sa valeur explicative sont les mêmes que celles d'une interprétation historique et c'est pourquoi de vouloir en faire une "loi * de l'évolution revient à introduire une forme d'historicisme en biologie; 2) la prériode du Postcript After Twenty Years de Objective Knowledge (1972) et de son «Autobiography» (1974) au cours de laquelle 
il considère le système théorique explicatif néo-darwinien formé plus spécifiquement par la conjonction de (h1), (h4) et (h5) comme une "quasi-tautologie" non testable dont le statut logique est celui d'une "vérité logique *; sa fonction et sa valeur explicative sont de nous faire comprendre le principe de certaines situations typiques et de guider la recherche ; c'est pourquoi, il considère ce système théorique comme un "programme métaphysique de recherche»; 3) la période de sa rétractation en 1977 au Darwin College au cours de laquelle il affirme que l'hypothèse évolutionniste a été bien testée et que la théorie de la sélection naturelle (la conjonction de (h4) et (h5)) est un énoncé probabiliste ayant été testé; sa fonction et sa valeur explicative demeurent toujours les mêmes, à savoir, celles d'un "programme de recherche" mais qui n'est plus métaphysique cette fois.

Nous avons vu qu'on peut attribuer la différence des propos de Popper sur l'hypothèse évolutionniste (h1) entre la première et les deux autres périodes, non pas à un changement d'idée mais à un changement de perspective. Dans Misère de l'historicisme (1956), Popper examine cette hypothèse non dans son usage scientifique mais dans son usage "para-scientifique " tel celui des historicistes qui s'en réclament pour justifier leur prétention à pouvoir dévoiler les "lois" de l'histoire humaine. C'est une contribution durable de Popper de montrer que la théorie de l'évolution ne se prête pas à ce genre d'utilisation. Il ne faut pas y voir une attaque contre l'usage scientifique de cette hypothèse car Popper y combat le "déterminisme* en histoire. Or, l'anti déterminisme défendu par Popper, fait partie intégrante de la conception scientifique de l'évolution qui est présentée comme le fruit du hasard et de la sélection.

La rétractation popperienne porte plutôt sur la seconde période où il considère l'usage scientifique de la conjonction de (h1), (h4) et (h5). Comme nous le savons, celui-ci change d'idée en ce qui concerne la testabilité et le statut logique de cette conjonction, mais non par rapport à sa fonction et sa valeur explicative. Si auparavant il la considérait comme un principe universel non testable et ayant la nature d'une "quasi-tautologie * maintenant il la considère comme une généralisation probabiliste ayant été testée pour un nombre de cas étonnant.

Nous savons que deux raisons motivaient Popper à considérer la conjonction de (h4) et (h5) comme un programme métaphysique de recherche : 1 . du fait de son caractère «quasi tautologique " et non testable et 2 . du fait qu'elle 
fournit une explication du seul principe de situation typique (des modèles artificiellement simplifiés de situations réelles). En ce qui concerne le premier point, Popper a rejoint les rangs de ceux qui ne considèrent pas l'explication en termes de sélection naturelle comme une explication circulaire. Même si c'est pour des raisons différentes, comme Gould, Sober et Rosenberg, Popper considère qu'on peut définir de façon indépendante des termes comme «aptitude", "adaptation" et "survie". Mais, il nous faut bien remarquer, comme Rosenberg, que les facteurs impliqués (anatomie, physiologie, comportement, environnement, etc.) sont si nombreux qu'on ne saurait penser à une définition générale. C'est pourquoi l'explication néo-darwinienne doit demeurer le plus souvent expost facto et une explication du seul principe de situations extrêmement simplifiées. Nous touchons ici à la seconde raison de Popper pour considérer cette explication comme "programme de recherche", c'est-à-dire non pas une explication mais un guide nous indiquant dans quelle direction chercher cette explication. L'explication néo-darwinienne ne permet que de simuler les situations pour en comprendre le principe mais nous serions incapables sur cette base de formuler des prédictions précises ou des explications précise du détail. Popper ne change pas d'idée sur la fonction et la valeur explicative de la théorie néo-darwinienne. Même si elle est en principe testable, il demeure très difficile de la tester.

Le revirement popperien a des conséquences certaines sur un autre aspect de sa pensée, à savoir, sur sa conception des programmes métaphysiques de recherche. Auparavant, Popper semblait penser qu'un programme de recherche ou une explication du seul principe de situation typique ne pouvait pas être testés. C'est pourquoi il considérait ces programmes de recherche comme nécessairement métaphysiques.

Ces programmes de recherche, écrit Popper (1982a:32), /../ n'ont habituellement pas encore le caractère de théories scientifiques testables. Ils peuvent devenir des théories scientifiques, mais alors il n'y a plus aucune raison de les appeler des programmes de recherche (nous soulignons).

Cependant, la théorie néo-darwinienne doit être considérée comme un programme de recherche testable. Pourquoi alors continuer de l'appeler un "programme de recherche " ? Parce qu'elle demeure malgré tout une explication ex post facto du seul principe de situation artificiellement simplifiée et elle demeure incapable de permettre des 
prédictions précises. C'est là une limitation de l'explication en biologie évolutionniste dont ne semble pas prendre conscience M. Ruse lorsqu'il érit (1977: 165) : "Je ne suis pas très certain de ce qu'il faut faire de cet argument car il me semble si injuste.".

Mais la présente recherche avait l'allure d'un véritable casse-tête dont il fallait assembler les morceaux éparses. À la différence du casse-tête cependant, nous ne disposions pas d'une image nous guidant dans l'assemblage des différentes pièces et nous permettant de «vérifier * après coup la justesse de notre reconstruction. C'est pourquoi, elle doit demeurer une interprétation possible de la position de Popper en biologie évolutionniste.

Faculté de philosophie Université Laval

\section{RÉFÉRENCES BIBLIOGRAPHIQUES}

Bouveresse, R. (1978), Karl Popper ou le rationalisme critique, Paris, Librairie philosophique J. Vrin.

GouLD, S.J. (1977), «Darwin's Untimely Burial » repris dans SOBER (ed.) (1984b) : 31-6.

HALSTEAD, Beverley (1980), " Popper : Good Philosophy, Bad Science?" dans New Scientist, Vol. 87, No. 1210, (17 juillet) : 215-7.

HAYEK, F. A. von (1967), Studies in Philosophy, Politics and Economics, Chicago, The University of Chicago Press.

KITTS, D.B. (1977), « Karl Popper, Verifiability and Systematic Zoology » dans Systematic Zoology, 26 : 185-94.

LEE, K.K. (1969) «Popper's Falsifiability and Darwin's Natural Selection " dans Pbilosopby, $44: 291-302$.

NADEAU, Robert (1985), "Popper, Hayek et la question du scientisme * Cabiers d'épistémologie, Montréal, Université du Québec à Montréal, Département de philosophie. Repris dans Manuscrito, IX, 2 (octobre 1986): 125-56. 
NADEAU, Robert (1983), «Problèmes philosophiques actuels du darwinisme "Cabiers d'épistémologie, No. 8306. Repris dans J. Lévy et $\mathrm{H}$. Cohen (dir.) Darwin après Darwin, Québec, Presses de l'Université du Québec, (1984) : 85-110.

POPPER, K.R. (1956), Misère de l'historicisme, Paris, Plon.

(1959) The Logic of Scientific Discovery, Londres, Routledge and Kegan Paul. Trad. anglaise de Logic der Forschung (1934). Nous utilisons l'édition anglaise (1968), New York/Evanston, Harper and Row («Harper Torchbooks»). Les traductions sont de nous.

(1972) Objective Knowledge. An Evolutionary Approach, Oxford, Clarendon Press. Nous utilisons l'édition revue (1983), Oxford, Clarendon Press. Les traductions sont de nous.

(1974) *Autobiography of Karl Popper» dans SCHII.PP (ed.).

(1974) : 1-156 et « Replies to my Critics : : 963-1196.

(1976) Unended Quest, Lasalle, Open Court.

(1978) *Natural Selection and the Emergence of Mind * dans Dialectica, Vol. 32, Nos. 3-4 : 339-55.

(1980) «Evolution. Lettters" dans New Scientist, Vol 87, No. $1211: 611$.

(1982a) The Open Universe. An Argument for Indeterminism, (Vol. II du Postscript) W.W. Bartley (ed.) Totowa/New Jersey, Rowman and Littlefield.

(1982b) Quantum Theory and the Schism in Pbysics (Vol. III. du Postscript) W.W. Bartley (ed.) Totowa/New Jersey, Rowman and Littlefield.

(1983) Realism and the Aim of Science (Vol. I. du Postscript) W.W. Bartley (ed.) Totowa/New Jersey, Rowman and Littlefield.

rosenberg, A. (1980) Sociobiology and the Preemption of Social Sciences, Baltimore, The John Hopkins Press.

(1985) The Structure of Biological Science, Cambridge, University Press. 
RusE, M. (1973) The Philosophy of Biology, London, Hutchinson.

(1977) «Karl Popper's Philosophy of Biology \# dans Philosophy of Science, 44: 638-61. Repris dans RUSE, M. (ed.) But is it Science? The Pbilosopbical Question in the Creation/Evolution Debate, Buffalo, Prometheus Books (1988) : 156-76.

SCHILPP, P.A. (ed.) (1974) The Philosopby of Karl Popper, Lasalle/llinois, Open Court (*The Library of Living Philosophers").

SOBER, Elliot (1984a) The Nature of Selection. Evolutionary Theory in Philosopbical Focus, Cambridge, Massachussett/London, England, The MIT Press ("A Bradford Book").

SOBER, Elliot (ed.) (1984b) Conceptual Issues in Evolutionary Biology, Cambridge, Mass., The MIT Press (*A Bradford Book").

WILLIAMS, M.B. (1973) « Falsifiable Predictions of Evolutionary Theory* dans Pbilosopby of Science, $40: 518-37$.

(1984) «The Logical Status of Natural Selection and Other Evolutionary Controversies» dans SOBER (ed.) (1984b) : 83-98. 